\title{
The association between tumour density and prostate cancer recurrence following radical prostatectomy
}

\author{
Luke T. Lavallée, MD; Rodney H. Breau, MD, FRCSC;, Mark A. Preston, MD; ; Gayanna Raju, MD; \\ Christopher Morash, MD, FRCSC,; Steve Doucette, MSc, ${ }_{i}^{+}$Ronald G. Gerridzen, MD, FRCSC; \\ James Eastham, MD,s Ilias Cagiannos, MD, FRCSC*
}

*Division of Urology, Department of Surgery, The Ottawa Hospital, University of Ottawa, Ottawa, ON; †Ottawa Hospital Research Institute, Ottawa, ON; §Department of Surgery, Urology Service, Memorial Sloan-Kettering Cancer Center, New York, NY

See related article on page 402.

Cite as: Can Urol Assoc J 2011;5(6):397-401; http://dx.doi.org/10.5489/cuaj.11061

\section{Abstract}

Purpose: Tumour density (TD) may be an independent prognostic factor in men with prostate cancer. The purpose of this study was to evaluate the association between prostate cancer TD and recurrence following radical prostatectomy.

Materials and Methods: Between 1995 and 2007, 645 patients from The Ottawa Hospital or Memorial Sloan-Kettering Cancer Center who had cancer and prostate volumes measured from radical prostatectomy specimens. Tumour density was defined as the relative tumour to prostate volume (tumour volume/prostate volume) and recurrence was defined as a prostate-specific antigen (PSA) $>0.2 \mathrm{ng} / \mathrm{mL}$ and rising, or postoperative use of radiation or hormonal therapy. Associations between TD and recurrence are adjusted for preoperative PSA, prostatectomy Gleason sum, tumour stage and margin status.

Results: Median follow-up was 40.8 months. Tumour density was associated with preoperative PSA, Gleason sum, tumour stage and surgical margin status (all $p<0.0001$ ). As a continuous variable, TD predicted recurrence-free survival (adjusted HR 1.34 per $10 \%$ increase in TD; $p=0.04$ ). As a categorical variable, the group of patients with a TD of $>10 \%$ had a 2.7 times greater hazard of recurrence compared to patients with a TD $<5 \%(95 \% \mathrm{Cl} 1.41$, 5.19; $p=0.003)$. Despite the independent association between TD and recurrence, the clinical value of TD remains in question as the discriminative performance (area under the curve) of predictive models only improved from 0.865 to 0.876 .

Conclusions: Prostate cancer TD is associated with known prognostic factors and is also independently predictive of recurrence following radical prostatectomy.

\section{Introduction}

Prostate cancer is the most common non-cutaneous malignancy in men. ${ }^{1}$ Radical prostatectomy (RP) is considered the gold standard for treating clinically localized cancer. ${ }^{2}$ Despite this, disease recurs in about $24 \%$ of men following RP for localized disease. ${ }^{3}$ Determining markers that predict recurrence is critical for patient counselling and treatment recommendations. Well-established prognostic factors following RP are pathologic stage, Gleason score (grade), preoperative prostate-specific antigen (PSA) and surgical margin (SM) status. ${ }^{4}$ These factors are used to determine prognosis, assess potential benefit of adjuvant therapy, and stratify patients in clinical trials. Other factors postulated as potential prognostic markers include: high-grade tumour volume, maximal tumour diameter, perineural invasion, DNA ploidy and molecular markers (E-cadherin, Ki-67, p53 expression). ${ }^{4,5}$ While many of these factors are associated with prognosis, they are not highly predictive of outcome after adjusting for stage, grade, PSA and SM status.

Tumour volume/size is independently associated with outcome in many malignancies, such as renal cell carcinoma. However, the prognostic value of tumour volume in prostate cancer is unclear since previous studies have inconsistent results. ${ }^{4,6-8}$ Prostate volume as a prognostic marker of prostate cancer following RP has also had variable outcomes, and thus neither tumour nor prostate volumes are routinely used for outcome prediction.

Tumour density (TD) is the tumour volume relative to the prostate volume (TV/PV), and can be thought of as the relative amount/percentage of an organ involved with cancer. Tumour density is grossly incorporated into the TNM staging system for prostate cancer in the form of pT2 substaging. The purpose of our study was to evaluate the association between TD and prostate cancer recurrence.

\section{Methods}

We obtained ethics review board approval at both research sites (The Ottawa Hospital and the Memorial Sloan-Kettering Cancer Center [MSKCC]), each of which is a tertiary care centre offering dedicated urologic oncology services. Data 
Lavallée et al.

from patients treated with RP were pooled from institutional databases. Available baseline information included age, pre-treatment PSA, RP Gleason sum, pathologic stage and SM status. Given the unknown significance of tumour and prostate volumes, these data were only reported by certain pathologists during certain years.

\section{Study sample}

Between 1995 and 2007, 444 patients were treated with RP by a uro-oncologist at The Ottawa Hospital. Of these, 213 had tumour and prostate volume measurements. At MSKCC, tumour volume was recorded sporadically between 2001 and 2004. Of the 2200 patients who underwent RP during this time period, 432 had tumour and prostate volume measurements. There were no known factors that influenced the decision to report tumour volume as it was reported at the discretion of the reviewing pathologist. Patients with lymph node metastases were excluded from the analysis.

\section{Pathologic analysis}

At The Ottawa Hospital and MSKCC, RP specimens were harvested in the operating room, inked to evaluate margin status and fixed in formaldehyde as per routine pathologic processing procedures. Prostate volume was measured by a water displacement method. Complete sampling using 3-mm slices on tissue slides were used to calculate the tumour volume by one of two previously validated methods in which our dedicated genitourinary pathologists had been trained, namely (1) constructed tumour maps or (2) by using the tumour dimensions and a correction factor derived from the equation for an ellipsoid structure. ${ }^{9-12}$ All tumour foci and all cancer grades were included in the calculation of tumour volume. RP Gleason sum, SM status, seminal vesicle invasion (SVI) and extraprostatic extension (EPE) were derived using standard pathological techniques.

\section{Statistical analysis}

Data were summarized and the associations between TD and potential confounding baseline variables were performed using linear or logistic regression as appropriate. Multivariable Cox proportional hazards analyses incorporating pre-treatment PSA, RP Gleason sum, stage and SM status were conducted to assess the association between TD and recurrence (primary endpoint). Recurrence was defined as a PSA $>0.2 \mathrm{ng} / \mathrm{mL}$ and rising or at the initiation of postoperative radiation or hormonal therapy. Patients were censored at the time of non-prostate cancer death or at last followup. Tumour volume was also analyzed for its associations with pathologic and recurrence endpoints in an identical fashion to TD.

\section{Results}

The mean age of men at the time of RP was 63.0 (standard deviation $=6.7$ ) years with a median PSA value of 5.5 (inter-quartile range [IQR] 4.2-7.8). Median follow-up was 3.43 years (IQR 2.0-4.9). Demographic and tumour pathologic characteristics were stratified by TD (Table 1). To assess for selection bias, we compared baseline characteristics of patients with volume measurements to those who did not have these measurements performed (Table 1).

Tumour density was associated with known prognostic factors in univariate analysis (Table 2). In multivariable analysis, as a continuous variable, TD was modestly associated with cancer recurrence (HR 1.34 per $10 \%$ increase in TD; $p=0.04)$. To potentially improve clinical application, TD was modeled as a categorical variable: low TD (0-5\%), intermediate TD (5.1-10\%), and high TD ( $\geq 10.1 \%)$. Patients with high TD were at significantly increased risk of recurrence compared to patients with low TD $(\mathrm{HR} 2.7 ; 95 \% \mathrm{Cl}$ $1.41,5.19) ; p=0.003$ ) (Table 3).

Tumor volume was also assessed for its association with pathologic outcomes and prostate cancer recurrence. In multivariate analysis, tumour volume was not found to be statistically significantly associated with the prostate cancer recurrence following RP (HR 1.43 95\% Cl 0.71, 2.87; $p=0.34)$. Complete results of this analysis are not presented for brevity and clarity.

The ability of TD to improve current methods of predicting recurrence in patients with organ-confined prostate cancer was assessed using a receiver operator characteristic (ROC) curve and an AUC analysis. Two curves were constructed (1) a base model using EPE, SVI, SM, and RP Gleason sum (AUC $=0.865$ ) and (2) an enhanced model including all the variables of the base model and the addition of TD (AUC $=0.876)$.

\section{Discussion}

Disease outcome prediction allows for proper stratification of patients in clinical trials and allows for a more informed approach to patient care. ${ }^{13}$ Currently, pre-treatment PSA, Gleason score, stage and SM status are accepted and used prognostic markers. ${ }^{5,7,14}$ In this analysis, we observed that TD was independently associated with prostate cancer recurrence, however, adding this characteristic to predictive models did not significantly improve discriminative accuracy (AUC 0.876 with TD vs. 0.865 without TD).

In previous studies, tumour volume and prostate volume have been inconsistently associated with prognosis when evaluated independently. Therefore, the prognostic role of these independent volumes has not been established. Studies focused on prostate cancer tumour volume have found larger tumours are associated with progression, however the rela- 


\begin{tabular}{|c|c|c|c|c|c|}
\hline \multirow{2}{*}{ Demographics } & \multicolumn{4}{|c|}{ Tumour and prostate volume data available } & \multirow{2}{*}{$\begin{array}{c}\text { Tumour or prostate volume not available } \\
\text { Overall }\end{array}$} \\
\hline & $<5 \%$ & $5-10 \%$ & $>10 \%$ & Overall & \\
\hline Sample (n) & 409 & 136 & 100 & 645 & 1999 \\
\hline \multicolumn{6}{|l|}{ Age } \\
\hline Mean (SD) & $63.5(6.6)$ & $61.5(6.9)$ & $62.7(6.8)$ & $63.0(6.7)$ & $63.4(7.3)$ \\
\hline Median [IQR] & $63.5[59.4,68.1]$ & $61.7[56,66]$ & $61.8[58,68]$ & $63[58.9,67.8]$ & \\
\hline \multicolumn{6}{|c|}{ Tumour density } \\
\hline Median [IQR] & $1.5(0.5-2.9)$ & $7.3(6.0-8.5)$ & $12.9(11.3-17.4)$ & $3.3[1.1,7.6]$ & - \\
\hline \multicolumn{6}{|l|}{ PSA } \\
\hline Mean (SD) & $5.79(3.69)$ & $7.21(4.2)$ & $10.1(11.6)$ & $6.8(6.0)$ & $6.3(4.8)$ \\
\hline Median (IQR) & $5.1[3.93,6.9]$ & $6.1[4.68,8.71]$ & $7.4[5.2,11.3]$ & $5.5[4.2,7.8]$ & - \\
\hline$<10$ & $372(91.0 \%)$ & $110(80.9 \%)$ & $71(71.0 \%)$ & $553(85.7 \%)$ & - \\
\hline $10-20$ & $33(8.1 \%)$ & $22(16.2 \%)$ & $20(20.0 \%)$ & $75(11.6 \%)$ & - \\
\hline$>20$ & $4(1 \%)$ & $4(2.9 \%)$ & 9 (9\%) & $17(2.6 \%)$ & - \\
\hline \multicolumn{6}{|c|}{ RP Gleason sum } \\
\hline $2-6$ & $207(51 \%)$ & $46(33.8 \%)$ & $21(21.2 \%)$ & $274(42.8 \%)$ & $850(45.7 \%)$ \\
\hline 7 & $180(44.3 \%)$ & $84(61.8 \%)$ & $58(58.6 \%)$ & $322(50.2 \%)$ & $909(48.8 \%)$ \\
\hline 8-10 & $19(4 / 7 \%)$ & $6(4.4 \%)$ & $20(20.2 \%)$ & $45(7.0 \%)$ & $103(5.5 \%)$ \\
\hline \multicolumn{6}{|l|}{ SM } \\
\hline Positive & $34(8.3 \%)$ & 27 (19.9\%) & 31 (31\%) & $92(14.3 \%)$ & $265(13.9 \%)$ \\
\hline Negative & 375 (91.7\%) & $109(80.2 \%)$ & $69(69 \%)$ & $553(85.7 \%)$ & $1637(86.1 \%)$ \\
\hline \multicolumn{6}{|l|}{ EPE } \\
\hline Positive & $34(8.3 \%)$ & $41(30.9 \%)$ & $50(50 \%)$ & $166(25.7 \%)$ & $443(23.4 \%)$ \\
\hline Negative & $335(81.9 \%)$ & $94(69.1 \%)$ & $50(50 \%)$ & $479(74.3 \%)$ & $1447(76.6 \%)$ \\
\hline \multicolumn{6}{|l|}{ SVI } \\
\hline Positive & $6(1.5 \%)$ & $6(4.4 \%)$ & $20(20 \%)$ & $32(5.0 \%)$ & $101(5.3 \%)$ \\
\hline Negative & 403 (98.5\%) & $130(95.6 \%)$ & $80(80 \%)$ & $613(95.0 \%)$ & $1791(94.7 \%)$ \\
\hline
\end{tabular}

tionship is often explained by differences in other prognostic characteristics. ${ }^{5,14-17}$ More recently, prostate volume has been studied to determine the association with outcome. It has been found that large prostates are more likely to be associated with organ confined tumours. However, similar to tumour size, the independent association between prostate size and prognosis has not been definitively established. ${ }^{6,18,19}$

In the current study, we define density as the proportional tumour to prostate volume. Our hypothesis was that the proportion of the prostate containing tumour may be more informative than either variable alone. For example a $2-\mathrm{cm}^{3}$ tumour in a 20-gram prostate may be more significant than the same volume of tumour inside a 60 -gram prostate. Previous authors have examined tumour density under the terms Percent Tumour Involvement, Percent Carcinoma, and Relative Tumour volume, and although the results have been promising, it has not been incorporated into predictive nomograms. $4,9,10,20,21$ Previous studies have reported that percent tumour involvement was associated with positive SMs, EPE, SVI and biochemical progression in multivariate analysis after adjusting for other variables including year of surgery, PSA, race, clinical stage, Gleason sum, SM, extracapsular extension and SVI. ${ }^{20}$ These findings were consistent with a similar study of over 1800 patients with organ confined (pT2) disease. ${ }^{9}$ Some have suggested evaluating TD as a categorical variable. ${ }^{9,20}$ One study found that every $5 \%$ increase in the percentage of carcinoma was associated with an $11 \%$ increase in recurrence. ${ }^{10}$ Unfortunately, all studies have not confirmed these findings as some have found that density is not independent of other prognostic variables. ${ }^{21,22}$ Currently, there is no consensus as to the importance of reporting tumour or prostate volume in RP specimens and this uncertainty was highlighted in a recent publication by the International Society of Urological Pathology (ISUP) Concensus Conference. ${ }^{22}$

Our study found TD to be independently associated with cancer recurrence adjusting for other currently accepted prognostic variables (pre-treatment PSA, Gleason score, tumour stage and SM status). When TD was evaluated as a categorical variable using the categories $0-5 \%, 5.1-10 \%$, $>10 \%$, patients in the highest density category $(>10 \%)$ demonstrated a greater than two-fold increase in the risk of recur- 
Lavallée et al.

\begin{tabular}{lcc}
\hline $\begin{array}{l}\text { Table 2. Tumour density analyzed for association with } \\
\text { known predictors of prostate cancer disease-free interval }\end{array}$ \\
\hline & \multicolumn{2}{c}{ Univariate analysis } \\
\cline { 2 - 3 } & OR (95\% Cl) & p-value \\
\hline Tumour density & 1 & 0 \\
High-grade Gleason sum & $1.11(1.07,1.16)$ & $<0.0001$ \\
Pre-treatment PSA* & $0.26(0.04)$ & $<0.0001$ \\
Surgical margin & $1.10(1.07,1.14)$ & $<0.0001$ \\
Extraprostatic extension & $1.13(1.09,1.17)$ & $<0.0001$ \\
Seminal vesicle invasion & $1.14(1.09,1.20)$ & $<0.0001$ \\
\hline FThis includes pre-treatment PSA, surgical margin, extraprostatic extension, seminal vesicle \\
invasion and high-grade Gleason score 7-10, using unadjusted univariate analysis. \\
*Note the association of TV and TD with PSA is demonstrated using a parameter estimate \\
and standard error per 1 unit increase in PSA. \\
OR: odds ratio; Cl: confidence interval; PSA: prostate-specific antigen. \\
\hline
\end{tabular}

rence when compared to the $0-5 \%$ group in multivariate analysis.

The purpose of a prognostic marker is to improve patient counselling, assist in decision-making regarding the use of adjuvant therapy and improve stratification of trial participants. Although TD is statistically significantly associated with recurrence independent of current prognostic variables, we are unsure if it adds clinical value as the discriminative performance (AUC) improvement is minimal. The accuracy in calibration of predictive models is difficult to assess. Therefore, at this time, we believe tumour and prostate volume should be routinely collected by pathologists at academic research centres to allow further long-term assessment of important outcomes, such as metastases and death. However, pending further study, we currently do not use TD for patient counselling.

Limitations of our study include a relatively short followup time of 40.8 months, the retrospective nature of our data, and the large number of patients for whom complete data was not available. The potential selection bias was partially assessed by comparing the characteristics of patients with volume data available to those who did not have this information available (Table 1). Based on this assessment, selection bias is unlikely to have had a large effect on the results.

\section{Conclusions}

Tumour density is associated with known prostate cancer prognostic factors. In addition, TD is independently predictive of cancer recurrence. Despite this, the discriminative accuracy of prediction models is not significantly improved by the addition of TD. Further study evaluating the association between TD and long-term prostate cancer outcomes is warranted.

\section{Competing interests: None declared.}

\begin{tabular}{|c|c|c|}
\hline \multirow{2}{*}{\multicolumn{3}{|c|}{$\begin{array}{l}\text { Table 3. Tumour density as a continuous and categc } \\
\text { variable predicting for recurrence-free survival whil } \\
\text { controlling for other known predictors using multiv } \\
\text { analysis* } \\
\text { Recurrence-free survival multivariate analysis }\end{array}$}} \\
\hline & & \\
\hline & HR (95\% Cl) & $p$-value \\
\hline \multicolumn{3}{|l|}{ Tumour density } \\
\hline \multicolumn{3}{|l|}{ Continuous } \\
\hline Per 1 unit & $1.03(1,1.06)$ & 0.04 \\
\hline Per 10 units & $1.34(1.01,1.78)$ & 0.04 \\
\hline \multicolumn{3}{|l|}{ Categorical } \\
\hline $0-5 \%$ & 1 & 1 \\
\hline $5-10 \%$ & $1.34(0.64,2.81)$ & 0.44 \\
\hline $10 \%$ & $2.7(1.41,5.19)$ & 0.003 \\
\hline \multicolumn{3}{|l|}{ Adjusted variables } \\
\hline PSA & $1.02(1,1.04)$ & 0.03 \\
\hline High-grade Gleason sum & $3.18(2.23,4.53)$ & $<0.0001$ \\
\hline Surgical margin & $2.52(1.38,4.57)$ & 0.003 \\
\hline Extraprostatic extension & $2.08(1.12,3.88)$ & 0.02 \\
\hline Seminal vesicle invasion & $1.4(0.64,3.04)$ & 0.4 \\
\hline
\end{tabular}

This paper has been peer-reviewed.

\section{References}

1. Jemal A, Siegel R, XU J, et al. Cancer statistics, 2010. CA Cancer J Clin 2010;60:277-300.

2. Rayala HJ, Richie JP. Radical prostatectomy reigns supreme. Oncology (Williston Park) 2009;23:863-7.

3. Greene KL, Meng MV, Ekkin EP, et al. Validation of the Kattan preoperative nomogram for prostate cancer recurrence using a community based cohort: results from cancer of the prostate strategic urological research endeavor (capsure). J Urol 2004;171:2255-9.

4. May M, Siegsmund $M$, Hammermann $F$, et al. Visual estimation of the tumor volume in prostate cancer: a useful means for predicting biochemical-free survival after radical prostatectomy? Prostate Cancer Prostatic Dis 2007;10:66-71.

5. Epstein Jl, Amin M, Boccon-Gibod L, et al. Prognostic factors and reporting of prostate carcinoma in radical prostatectomy and pelvic lymphadenectomy specimens. Scand I Urol Nephrol Suppl 2005;216:34-63.

6. Pierorazio PM, Kinnaman MD, Wosnitzer MS, et al. Prostate volume and pathologic prostate cancer outcomes after radical prostatectomy. Urology 2007;70:696-701.

7. Palisaar RJ, Graefen M, Karakiewicz PI, et al. Assessment of clinical and pathologic characteristics predisposing to disease recurrence following radical prostatectomy in men with pathologically organ-confined prostate cancer. Eur Urol 2002;41:155-61.

8. Wolters T, Roobol MJ, van Leeuwen PJ, et al. Should Pathologists Routinely Report Prostate Tumour Volume? The Prognostic Value of Tumour Volume in Prostate Cancer. Eur Urol 2009; Epub 2009 Jul 29.

9. Ramos $\mathrm{CG}$, Roehl KA, Antenor JA, et al. Percent carcinoma in prostatectomy specimen is associated with risk of recurrence after radical prostatectomy in patients with pathologically organ confined prostate cancer. J Urol 2004;172:137-40.

10. Carvalhal GF, Humphrey PA, Thorson P, et al. Visual estimate of the percentage of carcinoma is an independent predictor of prostate carcinoma recurrence after radical prostatectomy. Cancer 2000;89:1308-14.

11. Humphrey PA, Vollmer RT. Percentage carcinoma as a measure of prostatic tumor size in radical prostatectomy tissues. Mod Pathol 1997:10:326-33.

12. Mail KT, Mokhtar G, Burns BF, et al. A simple technique for calculation of the volume of prostatic adenocarcinomas in radical prostatectomy specimens. Pathol Res Pract 2003;199:599-604.

13. Bolla $M$, van Poppel $H$, Collette $L$, et al. Postoperative radiotherapy after radical prostatectomy: a randomised controlled trial (EORTC trial 22911). Lancet 2005;366:572-8. 
Prostate cancer tumour density

14. Salomon L, Levrel 0 , Anastasiadis AG, et al. Prognostic significance of tumor volume after radical prostatectomy: a multivariate analysis of pathological prognostic factors. Eur Urol 2003;43:39-44.

15. Chun FK, Briganti A, Jeldres $C$, et al. Tumour volume and high grade tumour volume are the best predictors of pathologic stage and biochemical recurrence after radical prostatectomy. Eur J Cancer 2007;43:536-43.

16. Kikuchi E, Scardino PT, Wheeler TM, et al. Is tumor volume an independent prognostic factor in clinically localized prostate cancer? J Urol 2004;172:508-11.

17. Nelson BA, Shappell SB, Chang SS, et al. Tumour volume is an independent predictor of prostate-specific antigen recurrence in patients undergoing radical prostatectomy for clinically localized prostate cancer. BJU Int 2006;97:1169-72.

18. Bianco FJ Jr, Mallah KN, Korets R, et al. Prostate volume measured preoperatively predicts for organconfined disease in men with clinically localized prostate cancer. Urology 2007;69:343-6.

19. Yadav R, Tu JJ, Jhaveri J, et al. Prostate volume and the incidence of extraprostatic extension: is there a relation? J Endourol 2009:23:383-6.

20. Rampersaud EN, Sun L, Moul JW, et al. Percent tumor involvement and risk of biochemical progression after radical prostatectomy. J Urol 2008; 180:571-6; discussion 576

21. Guzzo TJ, Vira MA, Neway W, et al. Minimal tumor volume may provide additional prognostic information in good performance patients after radical prostatectomy. Urology 2007;69:1147-51.

22. van der Kwast TH, Amin MB, Billis A, et al. International Society of Urological Pathology (ISUP) Consensus Conference on Handling and Staging of Radical Prostatectomy Specimens. Working group 2: T2 substaging and prostate cancer volume. Mod Pathol 2011;24:16-25.

Correspondence: Dr. llias Cagiannos, Division of Urology, B-327, The Ottawa Hospital - Civic Campus, 1053 Carling Ave., Ottawa, ON KIY 4E9; fax: 613-761-5305; icagiannos@ottawahospital.on.ca 